Revue d'histoire de l'Amérique française

REYUE D.HISTOIRE DE L'AMÉRIQUE FRANÇAISE

\title{
FAUCHER, Philippe et Johanne BERGERON, Hydro-Québec, la société de l'heure de pointe. Montréal, Les Presses de l’Université de Montréal, 1986. 221 p. 19,95 \$
}

\section{Claude Bellavance}

Volume 41, numéro 2, automne 1987

URI : https://id.erudit.org/iderudit/304572ar

DOI : https://doi.org/10.7202/304572ar

Aller au sommaire du numéro

Éditeur(s)

Institut d'histoire de l'Amérique française

ISSN

0035-2357 (imprimé)

1492-1383 (numérique)

Découvrir la revue

Citer ce compte rendu

Bellavance, C. (1987). Compte rendu de [FAUCHER, Philippe et Johanne BERGERON, Hydro-Québec, la société de l'heure de pointe. Montréal, Les Presses de l'Université de Montréal, 1986. 221 p. 19,95 \$]. Revue d'histoire de l'Amérique française, 41(2), 283-286. https://doi.org/10.7202/304572ar d'utilisation que vous pouvez consulter en ligne. 
FAUCHER, Philippe et Johanne BERGERON, Hydro-Québec, la société de l'heure de pointe. Montréal, Les Presses de l'Université de Montréal, 1986. 221 p. $19,95 \$$

Plusieurs ouvrages traitant de la grande société d'État québécoise ont paru ces dernières années ${ }^{1}$. L'étude des politologues Philippe Faucher et Johanne Bergeron est consacrée à la gestion et aux stratégies d'entreprise d'HydroQuébec depuis le second volet de l'étatisation de l'électricité (1962). Comment Hydro-Québec s'acquitte-t-elle de son double mandat, soit de fournir l'électricité nécessaire au Québec au meilleur compte possible et d'agir comme agent important de développement économique? Quel contrôle l'État a-t-il maintenu sur les grandes orientations de sa société de production et de distribution d'électricité au cours des vingt-cinq dernières années? Une telle diversité de pistes de recherche a donné lieu à un ouvrage quelque peu hétéroclite mais tout de même stimulant. Adoptant parfois un ton polémique, les auteurs présentent aussi une série de propositions visant à maximiser l'impact positif d'Hydro-Québec sur l'économie québécoise.

Le livre est divisé en deux grandes parties: «Hydro-Québec et les choix énergétiques du Québec», et «Hydro-Québec comme instrument de politique économique». Un premier chapitre, faisant corps avec l'introduction, précède

\footnotetext{
1 Voir entre autres, Clarence Hogue, André Bolduc et Daniel Larouche, Québec, un siècle d'électricité (Montréal, Éditions Libre Expression, 1979); Alain Chanlat et al., Gestion et culture d'entreprise: les cheminements d'Hydro-Québec (Montréal, Québec-Amérique, 1984).
} 
toutefois ces deux parties. On y traite de certains des problèmes fondamentaux que soulève l'étude d'une entreprise d'État. Appréhendée en tant qu'organisation et définie comme «un lieu de pouvoir et d'enjeux politiques où se réalise la conciliation entre les intérêts véhiculés par les forces du marché et ceux qui s'expriment à travers l'appareil d'État» (p. 26), l'entreprise publique profitera au maximum du laxisme de l'État en matière de politiques économiques pour établir ses propres critères de gestion. Plus les politiques économiques gouvernementales seront imprécises, plus grande sera l'autonomie de gestion et «l'espace d'intervention» de la société. En mettant à profit cet espace d'intervention pour accroître son indépendance, elle augmentera sa distance par rapport à l'Etat et, forcée de tenir compte de la logique du marché, elle sera portée à aligner sa stratégie d'entreprise sur celle des firmes du secteur privé. Au dire des auteurs, ces règles applicables à toute entreprise publique se vérifient clairement dans le cas d'Hydro-Québec. En effet, en l'absence de politique gouvernementale précise en matière d'énergie, Hydro-Québec aurait mis de l'avant une stratégie de commercialisation de l'énergie électrique et un programme d'investissement ne reposant pas prioritairement sur les intérêts de la collectivité. En tant qu'organisation, l'entreprise géante de production et de distribution de l'électricité défend tout d'abord ses propres intérêts.

Après un bref survol de ce qu'ils ont nommé «la nationalisation tronquée de $1962 »^{2}$, P. Faucher et J. Bergeron passent en revue les grandes réalisations d'Hydro-Québec des années 1960 et 1970; c'est l'ère des grands projets qui, à l'exception de l'expérience plutôt malheureuse dans le domaine du nucléaire, apparaissent comme autant de belles réussites aux plans technique et organisationnel. Mais, mettre en chantier des aménagements hydro-électriques de l'envergure du complexe de la Baie James suppose que l'on dispose d'un marché suffisamment développé pour écouler toute cette énergie lorsqu'elle deviendra disponible. La prévision de la demande, on le comprend, est au centre des préoccupations de toute entreprise d'électricité. L'analyse de la méthode prévisionnelle utilisée par Hydro-Québec au cours des vingt dernières années amène les auteurs à critiquer la gestion du patrimoine hydraulique collectif pratiquée par la société d'État. Une telle gestion entraînerait un gaspillage de nos ressources: c'est «la tyrannie de l'heure de pointe». En effet, comme on ne peut stocker massivement l'énergie électrique produite par les centrales, il faut, pour répondre au premier mandat d'Hydro-Québec - fournir l'électricité aux Québécois -, ajuster la capacité du réseau à la demande de pointe $^{3}$. Les grandes décisions d'investissement sont donc prises en fonction d'une estimation de ce que sera l'appel maximal d'électricité dans les années à venir. Jusqu'à très récemment, la méthode de prévision de la demande d'électricité reposait sur une base hautement empirique. Il s'agissait essentiellement d'appliquer les taux de croissance des années antérieures tout en considérant un nombre limité de facteurs externes. Or cette méthode, appropriée jusqu'au début des années 1970, s'est révélée de moins en moins utilisable par

2 En 1962, près de 40\% de la puissance installée au Québec demeure la propriété de quelques firmes industrielles auto-productrices. La plus importante est Alcan qui dispose des sites les plus avantageux du bassin hydrographique saguenayen.

3 La demande maximale annuelle est normalement enregistrée au plus fort de l'hiver. Mentionnons au passage que la compréhension des termes techniques et des indicateurs financiers est grandement facilitée par un lexique bien construit présenté en annexe. 
la suite. Au moment où, forcée par l'État, Hydro-Québec se mit à employer l'économétrie à cette fin, l'entreprise se trouvait déjà aux prises avec des surplus considérables d'énergie électrique à écouler.

La fin des années 1970 et le début des années 1980 marquent un tournant décisif en ce qui concerne la stratégie d'entreprise d'Hydro-Québec. Des difficultés financières l'incitent à augmenter considérablement ses tarifs d'électricité. Elle remet alors en question la majeure partie de son programme d'investissement pour les vingt prochaines années et s'efforce de commercialiser l'énergie excédentaire, consentant notamment d'importantes baisses de tarif à certaines entreprises industrielles grandes consommatrices. La société se tourne résolument vers le marché des exportations et propose une série de programmes d'économie d'énergie. Mais, au dire des auteurs, «la stratégie d'HydroQuébec apparaît singulièrement inadaptée: dans un contexte d'équilibre de l'offre et de la demande où se profilent des perspectives de pénurie [début des années 1970], l'entreprise entreprend de stimuler la demande, alors que dans le contexte de surplus [après la mise en service des centrales de la Baie James], elle hausse ses tarifs et parle d'économie d'énergie et de rationalisation de la consommation» (p. 119). La firme s'était appuyée sur une hausse continue du volume des ventes; désormais sa croissance sera garantie principalement par des hausses de tarifs. Hydro-Québec disposerait pourtant d'une marge de manoeuvre suffisante pour accroître considérablement son impact sur le développement économique du Québec sans pour autant remettre en cause sa crédibilité auprès des bailleurs de fonds. C'est du moins ce qui semble ressortir de l'étude financière de la société d'État. Les résultats comparés d'HydroQuébec et d'Hydro-Ontario laissent entrevoir des ratios financiers hautement favorables à la société d'État québécoise; et pourtant, la cote de crédit allouée par des firmes américaines spécialisées s'avère nettement moins élevée ${ }^{4}$. Ce sont les cotes des provinces respectives elles-mêmes qui expliqueraient ce curieux décalage, une société publique ne pouvant, semble-t-il, se voir attribuer une note supérieure à celle de l'État qui s'en porte garant. Dans de telles conditions, la gestion d'Hydro-Québec apparaît beaucoup trop «conservatrice» et impliquerait un gaspillage de ressources. Si le maintien de tels ratios favorise l'indépendance de l'entreprise par rapport à l'État, il n'en constitue pas moins une pratique fort coûteuse pour la collectivité. Le message ici est clair: qu'Hydro-Québec se comporte comme les autres firmes du même secteur dotées des cotes de crédit équivalentes, elle pourra alors reprendre un rythme de croissance satisfaisant. Dans l'esprit de P. Faucher et de J. Bergeron, il incombe à l'État québécois d'occuper la place qui lui revient et de faire en sorte que sa société modifie sa stratégie d'entreprise.

Instructif sur des thèmes comme la tarification, sur l'importance économique d'Hydro-Québec - qui demeure le principal investisseur public ou privé de la province - , sur l'autonomie de gestion laissée aux administrateurs par les organismes gouvernementaux et sur la politique d'achat pratiquée par

4 «Aaa» pour Hydro-Ontario, soit la cote la plus haute qu'une entreprise puisse obtenir, et «A1» pour Hydro-Québec, résultat à peine supérieur à «Bbb». On sait qu'à toutes fins utiles, cette cote détermine les taux d'intérêt payés sur les emprunts substantiels contractés sur le marché financier américain. 
cette société, l'ouvrage comporte néanmoins des faiblesses sur le plan de la cohérence interne. A notre avis, les auteurs n'ont pu éliminer toute ambiguïté quant à leur objet d'étude. En introduction, on annonce tour à tour que la société d'État sera considérée en tant qu'organisation, agent économique à part entière et lieu de pouvoir. Un peu plus loin, on affirmera successivement que l'ouvrage est consacré à l'examen du revirement de la stratégie d'HydroQuébec, et que la question fondamentale n'est pas le contrôle, les jeux d'influence et les luttes de pouvoir entre les hommes politiques et les administrateurs de la société, mais que «le problème est celui de la responsabilité dans la définition des orientations et des moyens d'action» (p. 15-16). On s'interroge enfin à savoir si «l'entreprise publique peut servir d'instrument de politique économique». C'est là, ajoute-t-on, «la question centrale qui traverse cet ouvrage». Ce que $\mathrm{P}$. Faucher et J. Bergeron n'hésitent pas à qualifier de «cadre théorique» (p. 117) se révèle largement insuffisant et plutôt mal intégré. La division en deux parties correspondant aux deux mandats d'Hydro-Québec et, plus généralement, le plan de l'ouvrage susciteront également des réserves. Les auteurs n'ont pu éviter certaines redites, et la plupart des chapitres - dont certains avaient déjà paru sous forme d'articles - vivent encore trop pour euxmêmes et pas assez en fonction de l'ensemble. De plus, à notre avis, les chapitres sur la nationalisation et sur les grands projets des années 1960 et 1970 n'apportent à peu près rien de neuf et auraient gagné à être fusionnés en un seul. Ces réserves émises, l'ouvrage n'en demeure pas moins fort intéressant pour quiconque désire saisir l'évolution de la stratégie de cette grande entreprise québécoise depuis une vingtaine d'années, de même que celle de ses rapports avec l'État. 